\section{Nothing in between: a multi-faith response to the paper on religion and suicide}

As a Liberal Jewish psychiatry registrar and a moderately observant Hindu psychiatry senior house officer, we read with great interest Kleiman \& Liu's fascinating paper on the relationship between religious service attendance and suicide risk. ${ }^{1}$ We were also pleased to note that the paper has already generated sufficient interest to give rise to a fascinating editorial by $\mathrm{Cook}^{2}$ as well as helpful correspondence between the authors and Professor Nebhinani. ${ }^{3}$ This seems to attest to the importance of this topic, and we hope that our additional reflections on the methodology of Kleiman \& Liu's study can be part of an evolving dialogue around the interactions between mental health and religion/spirituality.

First, while there are many advantages to the prospective study design, difficulties are produced when the outcome of interest (here, completed suicide) is a relatively rare one. Only 25 completed suicides occurred, and the absolute numbers occurring in the two groups (frequent $v$. less frequent service attenders) were not specified. Even a very small swing in the distribution of the suicides from one group to the other could significantly alter the apparent magnitude of the protective effect of service attendance.

Second, the absence of any intermediate data between baseline and the end of the study period makes it difficult to draw conclusions about the potential link between religious service attendance and suicide - hence the title of our letter, 'Nothing in between'. During the follow-up period (12-18 years), much might have changed in people's lives, behaviours and health. In particular, people's level of religious observance (in the form of service attendance) might well have varied over the study period - as might their mental health. Moreover, there might well be interactions between these two variables. With only two datapoints (baseline self-report and a dichotomous outcome of suicide/not-suicide), it is impossible to know people's religiosity and mental health across the study period.

Third, this lack of intermediate data might stem from the fact that Kleiman \& Liu's study seems to have 'piggy-backed' onto a separate, pre-existing epidemiological survey, ${ }^{4}$ the primary objective of which was not the investigation of the relationship between religiosity and suicide. Convenient as it might have been to make use of pre-existing data, it might be that a study set up specifically to address the research question would offer richer information and allow greater extrapolation and clinical application.

Fourth, and also in terms of clinical applicability, we would question whether the focus on completed, as opposed to attempted, suicide is necessarily an advantage. As the authors observe, there do seem to be differences between the clinical profiles of those who complete, compared with attempt, suicide. However, the two are closely related, with previous suicide attempts a sufficiently well-recognised risk factor for suicide completion that it has been controlled for as a potential confounder in Kleiman \& Liu's study, even though it was not found to be 'a significant predictor of death by suicide'. Moreover, in the clinical setting, suicide attempts are one of the primary risk events of concern, but the study does not provide information on how religious service attendance might relate to these - information which could be of considerable relevance for risk assessment.

Given the above reflections, as well as the study limitations identified by Kleinman \& Liu, Cook and Nebhinani, we caution against an over-simplistic reading of the article's headline finding. In our clinical experience, the relationship between a person's religiosity and their risk of self-harm/suicide can vary considerably. We therefore urge that clinicians continue to conduct detailed explorations of each patient's individual dynamic risk factors and not overly focus on particular population-level static risk factors.

1 Kleiman EM, Liu RT. Prospective prediction of suicide in a nationally representative sample: religious service attendance as a protective factor. Br J Psychiatry 2014; 204: 262-6.

2 Cook CCH. Suicide and religion. Br J Psychiatry 2014; 204: 254-5.

3 Nebhinani N. Religious service attendance as a protective factor against suicide (letter). Br J Psychiatry 2014; 204: 404.

4 National Center for Health Statistics (US). Plan and operation of the Third National Health and Nutrition Examination Survey, 1988-1994. Vital Health Stat 1 1994; 32: 1-407.

Samuel C. Yates, Psychiatry Specialist Registrar, West London Mental Health Trust, UK. Email: samuelyates@doctors.org.uk; Donna Arya, Psychiatry Senior House Officer, West London Mental Health Trust, UK.

doi: 10.1192/bjp.205.2.163

Authors' reply: While we agree with several of the points raised by Yates \& Arya, further discussion of several issues is needed. First, we agree that caution is needed when interpreting prediction of a low base-rate occurrence such as suicide. As we note in the paper, although suicide is an infrequently occurring event, the number of suicides in the data-set matches what would be expected from the population during the study period. ${ }^{1}$ Considerable effort was taken by the United States Centers for Disease Control and Prevention to guarantee high accuracy of the cause of death data. The authors also noted that 'the absolute numbers occurring in the two groups (frequent $v$. less frequent service attendees) were not specified.' This information is available in Table 1 in the paper. Of the 25 people who died by suicide, $8(32 \%)$ attended religious services frequently and the other 17 did not.

Second, our outcome variable (death by suicide) was timevarying (i.e. our analyses examined religious service attendance not only as a predictor of death by suicide but also time to this event, which would differ from decedent to decedent). We agree that intermediate data in between the time an individual reported on their religious service attendance and the end of the study or their death would be desirable. It is important, however, to consider the feasibility of conducting such a multi-wave study with a large enough sample for meaningful analysis of a lowbase-rate event, such as death by suicide, as the outcome of interest. Additionally, we believe it is particularly telling that our measure of religious service attendance was able to predict suicide deaths in some cases several years later, despite any intermediate life changes. This might be because of the fact that a large number of participants $(42 \%)$ in the data-set were over 50: frequency of religious service attendance tends to be relatively stable in that age group. ${ }^{2}$ Thus, religious service attendance might not have varied much over the course of the study.

Third, as suggested by Yates \& Arya, collecting data on individual ( $v$. population level) time-varying prospective predictors of suicide might be ideal. Such a study, however, would be extremely expensive and resource intensive. Indeed, over 20000 people were needed for a data-set that had 25 suicides and data-sets such as this are rare. The number of participants needed to test the hypotheses in a fashion suggested by Yates \& Arya would be quite substantially more, and the related resources needed to conduct such a study would be magnified with each follow-up assessment.

Fourth, Yates \& Arya stated that using suicide deaths as an outcome variable might not be an advantage relative to using attempted suicide, in part because of the greater relevance of the latter to clinical settings. We caution against this view for several 
reasons. First, both deaths by suicide and suicide attempts are important public health concerns. Although a prior history of suicidal behaviour is indeed a strong predictor of future suicidal behaviour, a very large proportion of suicide deaths occur with the first attempt. Second, as cited in our manuscript, research finds that although suicide attempters and suicide decedents are overlapping groups, there is still a considerable lack of overlap between the two groups. ${ }^{3}$ Over $90 \%$ of people who attempt suicide do not go on to die by suicide. ${ }^{4}$ Moreover, several studies have assessed religion as a predictor of suicide attempts ${ }^{5}$ and thus our focus on death by suicide builds upon this literature.

1 Kleiman EM, Liu RT. Prospective prediction of suicide in a nationally representative sample: religious service attendance as a protective factor. Br J Psychiatry 2014; 204: 262-6.
2 Schwadel P. Age, period, and cohort effects on US religious service attendance: The declining impact of sex, southern residence, and Catholic affiliation. Sociol Relig 2010; 71: 2-24.

3 DeJong TM, Overholser JC, Stockmeier CA. Apples to oranges? A direct comparison between suicide attempters and suicide completers. J Affect Disord 2010; 124: 90-7.

4 Owens D, Horrocks J, House A. Fatal and non-fatal repetition of self-harm systematic review. Br J Psychiatry 2002; 181: 193-9.

5 Dervic K, Oquendo MA, Grunebaum MF, Ellis S, Burke AK, Mann JJ. Religious affiliation and suicide attempt. Am J Psychiatry 2004; 161: 2303-8.

Evan M. Kleiman, MA, Department of Psychology, George Mason University, VA, USA. Email: ekleiman@gmu.edu; Richard T. Liu, PhD, Department of Psychiatry and Human Behaviour, Brown University Alpert Medical School, Bradley Hospital, RI, USA.

doi: 10.1192/bjp.205.2.163a

\section{Correction}

Is depression one thing or many (letter)? BJP, 204, 488. The second author is: A. Odone. The online version of this letter has been corrected post-publication, in deviation from print and in accordance with this correction.

doi: $10.1192 / b j p .205 .2 .164$

\section{Retraction}

Childhood maltreatment and methylation of the glucocorticoid receptor gene $N R 3 C 1$ in bipolar disorder. BJP, 204, 30-35. An investigation carried out at the request of the Dean of the Faculty of Medicine of the University of Geneva has concluded that one of the authors (Alain Malafosse) fabricated methylation data. A reanalysis of the DNA reveals no significant correlation between childhood trauma and methylation of the NR3C1 gene. The original conclusions therefore no longer hold true and we wish to retract the paper.

J. M. Aubry, A. Dayer, N. Perroud, C. Piguet, A. Nallet, S. Favre

doi: 10.1192/bjp.205.2.164a 